\title{
Consenso Cultural sobre Alimentos en Adolescentes Embarazadas de Guadalajara, México
}

\author{
Cultural consensus regarding food in pregnant adolescents from \\ Guadalajara, México
Claudia C. Herrera-Suárez ${ }^{1}$, Javier E. García-De Alba², Edgar M. Vásquez-Garibay ${ }^{1}$, Enrique Romero-Velarde ${ }^{1}$, Hiliana P. Romo-Huerta ${ }^{3}$ y
Rogelio Troyo-Sanromán ${ }^{1}$

1 Instituto de Nutrición Humana, Universidad de Guadalajara. Jalisco, México.
2 UISESS-IMSS. Guadalajara, Jalisco, México. javier_91046@yahoo.com
3 Hospital Civil Dr. Juan I. Menchaca Guadalajara Jalisco, México.
Recibido 7 de Mayo 2008/Enviado para Modificación 25 de Octubre 2008/Aceptado 10 Noviembre 2008

RESUMEN

Objetivo Caracterizar la estructura semántica y el nivel de consenso cultural que manifiestan las adolescentes embarazadas que acuden a la consulta externa del Hospital Civil de Guadalajara Dr. Juan I Menchaca.

Métodos Estudio cualitativo mediante entrevistas estructuradas con la técnica de listas libres. Muestreo propositivo no aleatorizado que incluyo a 54 adolescentes embarazadas de 12 a 19 años de edad.

Resultados Los alimentos del desayuno y la cena obtuvieron un mayor grado de consenso cultural, detectándose 14 alimentos para el desayuno y 8 para la cena. Conclusión Conocer el modelo de consenso cultural de los alimentos nos permite reforzar, modificar o agregar alimentos saludables a la dieta de las adolescentes embarazadas.

Palabras Clave: Ingestión de alimentos, embarazo, características culturales (fuente: DeCS, BIREME).

\section{ABSTRACT}

Objective Characterising the semantic structure and cultural consensus level of pregnant adolescents attending outpatient consultation at the "Dr. Juan I Menchaca" civil hospital in Guadalajara.

Methods A qualitative study using free-list structured interviews. Non-randomised propositive sampling included 54 pregnant adolescents aged 12-19.

Results There was a significant degree of cultural consensus concerning breakfast and dinner food; 14 foods were detected for breakfast and 8 for dinner.

Conclusion Ascertaining the pattern of cultural consensus concerning food led us to reinforcing, modifying or adding healthy foods to pregnant adolescents' diet.

Key Words: Nutritional status, adolescent, pregnancy, culture (source: MeSH, NLM). 
n el campo de la promoción de salud, se ha desarrollado un interés
creciente por relacionar el proceso cognitivo de las personas, con
sus respuestas conductuales.

La antropología cognitiva como disciplina científica se ha preocupado por estudiar la incorporación del conocimiento compartido culturalmente entre diferentes grupos sociales (1). Para el efecto ha desarrollado estrategias que rescatan la subjetividad, mediante el lenguaje hablado aplicando la técnica de la entrevista en sus diversas modalidades (2).

Se trata, bajo la mirada del paradigma "emic" (donde se imponen las categorías de las personas investigadas) de evidenciar la cultura, definida como conocimiento compartido de una realidad concreta (3). Conocimiento que se incorpora a través de la experiencia interiorizada, y que se le dota de significados, que ayudan u orientan a las personas en la estructuración de dominios y modelos culturales, como marcos interpretativos con sentido para el desarrollo de sus acciones (4).

Al enfocar nuestra investigación desde los métodos de la antropología cognitiva, este dominio como conocimiento compartido culturalmente se puede rescatar a manera de modelo(s) cultural(es) que incluyen estructuras semánticas, (taxonomías de ítems), nivel de consenso y conocimiento cultural.

La caracterización de las representaciones culturales (5), puede ser un valioso complemento para los profesionales del área de la salud para reforzar, modificar, agregar el consumo de alimentos en la prescripción o programación nutrimental, en este caso para el grupo de las adolescentes embarazadas, donde las consecuencias de una mala nutrición:

1. Pueden afectar adversamente el desarrollo fisiológico tanto de la madre como de su hijo, debido a que pertenecen a dos grupos vulnerables de riesgo nutricio $(6,7)$, que en el caso de las adolescentes embarazadas, es trascendente como problema de salud, por la vulnerabilidad nutricia que manifiestan las mujeres en el periodo de la adolescencia y durante el embarazo (8-10).

2. Afectan los procesos psico-culturales, como el de identidad del yo de las adolescentes, debido a que un determinado tipo de alimentación les permite formar o no parte de un grupo, así como canalizar estrés y ansiedad (11), e inclusive cuestionarse su apariencia física y estilo de vida (12). 
Obtener conocimiento que integra las creencias de la gente sobre su alimentación, requiere realizarlo de manera sencilla, natural, espontánea y sin perder rigor científico, de tal forma que se evidencie sin obstáculos la cultura del grupo estudiado, sobre esta área concreta de la realidad, en este caso, nuestro propósito es caracterizar el dominio cultural y la estructura semántica de alimentos preferidos durante los diferentes tiempos de comida que constituyen la alimentación cotidiana de las adolescentes embarazadas que acuden a la consulta externa de un hospital público.

\section{MATERIAL Y MÉTODOS}

El universo de trabajo lo constituyeron las adolescentes embarazadas asistentes a la consulta externa del Hospital Civil de Guadalajara "Dr. Juan I Menchaca” (HCG) durante los meses de marzo a junio del año 2005. Se incluyeron adolescentes embarazadas aparentemente sanas, cuyas edades fluctuaran entre 12 a 19 años de edad, que aceptaron informadamente participar en el estudio.

Se trabajo con una muestra propositiva, de 54 personas, cuyo tamaño fue calculado con error de $1 \%$ y una potencia de $95 \%$, que duplica las recomendaciones de Weller (13) para muestras donde el conocimiento compartido a estimar es igual o superior a $50 \%$.

La captación de los datos se realizó en un sitio con privacidad; respetando los principios para realizar análisis de consenso cultural (3) que son:

a. Un dominio cultural unificado (los alimentos ingeridos para los diferentes tiempos de alimentación cotidiana).

b. Preguntas estructuradas y simples, en este caso en base a la técnica de las listas libres (14)(por ejemplo: ¿Dime cuáles son los alimentos que diariamente tomas para desayunar?).

c. Respuestas individuales e independientes. El proceso de los datos para el análisis de consenso cultural, se apoyó en el software AnthropacÒ (15), que realizó análisis competencial, para obtener la estructura semántica, nivel de conocimiento cultural del grupo, consenso cultural alrededor de uno o varios modelos semánticos de conocimiento en relación a los nutrimentos ingeridos según el tiempo de comida estudiado (desayuno, comida y cena). 
El análisis de los datos buscó dar respuesta a la existencia de un solo modelo semántico en el dominio cultural de los alimentos ingeridos según tiempo de comida. Para ello se determinó una razón de variabilidad mayor o igual a tres veces mayor del modelo considerado sobre los demás modelos obtenidos (3) $(\mathrm{p}<0.05)$.

\section{RESULTADOS}

Para el tiempo del desayuno (Tabla 1), solo se señalaron 14 alimentos por más de $10 \%$ de las adolescentes estudiadas, siendo el tiempo de alimento que mostró el mayor consenso, además de ser estadísticamente significativo $(\mathrm{p}<0.05)$, situación que apunta hacía la existencia de un solo modelo cultural compartido para el dominio semántico de los alimentos en el desayuno de las adolescentes embarazadas.

Tabla 1. Estructura semántica de los principales alimentos consumidos en el desayuno

\begin{tabular}{|c|c|c|c|}
\hline Lugar & Alimento & Número de Menciones & Porcentaje \\
\hline 1 & Leche & 31 & 57 \\
\hline 2 & Huevo & 26 & 48 \\
\hline 3 & Frijoles & 17 & 31 \\
\hline 4 & Pan dulce & 16 & 30 \\
\hline 5 & Hojuelas de maiz & 13 & 24 \\
\hline 6 & Licuado de leche con fruta & 12 & 22 \\
\hline 7 & Jugo de naranja & 9 & 17 \\
\hline 8 & Yogurt & 8 & 15 \\
\hline 9 & Refresco & 7 & 13 \\
\hline 10 & Рapaya & 6 & 11 \\
\hline 11 & $\begin{array}{l}\text { Hojuclas de maiz } \\
\text { azucaradas }\end{array}$ & 6 & 11 \\
\hline 12 & Frijoles refritos & 6 & 11 \\
\hline 13 & Galletas & 6 & 11 \\
\hline 14 & Manzana & 6 & 11 \\
\hline \multicolumn{4}{|c|}{ Analisis de Consenso } \\
\hline $\begin{array}{l}\text { Modelos } \\
\text { considerados }\end{array}$ & $\begin{array}{l}\text { Varianza } \\
\text { Estimada }\end{array}$ & $\begin{array}{c}\text { \% de la varianza total } \\
\text { cubierto por cada } \\
\text { modelo }\end{array}$ & $\begin{array}{l}\text { Razón de } \\
\text { Variabilidad }\end{array}$ \\
\hline $1^{\circ}$ & 15,01 & 65.9 & 3.56 \\
\hline $2^{a}$ & 4,2 & 18,5 & 1,18 \\
\hline $3^{\circ}$ & 3,5 & 15,6 & \\
\hline \multicolumn{4}{|c|}{ Promedio de Conocimiento Grupal $=0,30+\gamma=0,316$} \\
\hline
\end{tabular}

Los alimentos de alto contenido proteínico como la leche y el huevo ocuparon, los dos primeros lugares, seguidos de leguminosas, cereales y frutas. Las hojuelas de maíz con y sin azúcar, el yogurt y el refresco señalados como alimentos, ocuparon el $5^{\circ}, 11^{\circ}, 8^{\circ}, 9^{\circ}$ lugar entre las menciones, situación que contrasta con la ausencia de verduras para este tiempo de la alimentación cotidiana. 
La comida (Tabla 2) obtuvo un número mayor de menciones (10) por más de $10 \%$ de las adolescentes estudiadas, pero no se detectó consenso cultural en su consumo ( $\mathrm{p}>0.05)$.

Tabla 2. Estructura semántica de los principales alimentos consumidos en la comida

\begin{tabular}{|c|c|c|c|c|}
\hline Lugar & \multicolumn{2}{|c|}{ Alimento } & Número de Menciones & Porcentaje \\
\hline 1 & \multicolumn{2}{|c|}{ Carne } & 31 & 57 \\
\hline 2 & \multicolumn{2}{|c|}{ Agua fresca } & 26 & 48 \\
\hline 3 & \multicolumn{2}{|c|}{$\begin{array}{l}\text { Rebanadas de } \\
\text { papa fritas }\end{array}$} & 24 & 44 \\
\hline 4 & \multicolumn{2}{|c|}{ Refrescos } & 21 & 39 \\
\hline 5 & \multicolumn{2}{|c|}{ Caldos } & 20 & 37 \\
\hline 6 & \multicolumn{2}{|c|}{ Pollo } & 19 & 35 \\
\hline 7 & \multicolumn{2}{|c|}{ Pastas } & 16 & 30 \\
\hline 8 & \multicolumn{2}{|c|}{ Papa } & 14 & 26 \\
\hline 9 & \multicolumn{2}{|c|}{ Tortilla } & 13 & 24 \\
\hline 10 & \multicolumn{2}{|c|}{ Mango } & 11 & 20 \\
\hline 11 & \multicolumn{2}{|c|}{ Zanahoria } & 11 & 20 \\
\hline 12 & \multicolumn{2}{|c|}{ Frijoles } & 10 & 19 \\
\hline 13 & \multicolumn{2}{|c|}{ Sopa de arroz } & 9 & 17 \\
\hline 14 & \multicolumn{2}{|c|}{ Mole } & 8 & 15 \\
\hline 15 & \multicolumn{2}{|c|}{ Ensalada } & 8 & 15 \\
\hline 16 & \multicolumn{2}{|c|}{ Calabaza } & 8 & 15 \\
\hline 17 & \multicolumn{2}{|c|}{ Melón } & 7 & 13 \\
\hline 18 & \multicolumn{2}{|c|}{ Chayote } & 7 & 13 \\
\hline 19 & \multicolumn{2}{|c|}{ Manzana } & 7 & 13 \\
\hline 20 & \multicolumn{2}{|c|}{ Atún } & 6 & 11 \\
\hline \multicolumn{5}{|c|}{ Análisis de Consenso } \\
\hline \multicolumn{2}{|c|}{$\begin{array}{l}\text { Modelos } \\
\text { considerados }\end{array}$} & $\begin{array}{l}\text { Varianza } \\
\text { estimada }\end{array}$ & $\begin{array}{c}\% \text { de la varianza total } \\
\text { cubierto por cada } \\
\text { modelo }\end{array}$ & $\begin{array}{l}\text { Razón de } \\
\text { Variabilidad }\end{array}$ \\
\hline \multicolumn{2}{|c|}{$1^{\circ}$} & 10,4 & 60,3 & 2,3 \\
\hline \multicolumn{2}{|c|}{$\begin{array}{l}1^{\circ} \\
2^{\circ}\end{array}$} & 4,5 & 26,2 & 1,9 \\
\hline \multicolumn{2}{|c|}{$3^{\circ}$} & 2,3 & 13,5 & \\
\hline
\end{tabular}

Y aunque la carne ocupo el primer lugar entre las menciones, las frituras, los refrescos y los caldos ocuparon los lugares $3^{\circ}, 4^{\circ}$ y $5^{\circ}$ en el dominio semántico explorado.

El tiempo de la cena (Tabla 3), evidenció consenso cultural significativo $(\mathrm{p}<0.05)$ y se constituyó por leche, cereales, leguminosas, huevo y carne. 
Tabla 3. Estructura semántica de los principales alimentos consumidos en la cena

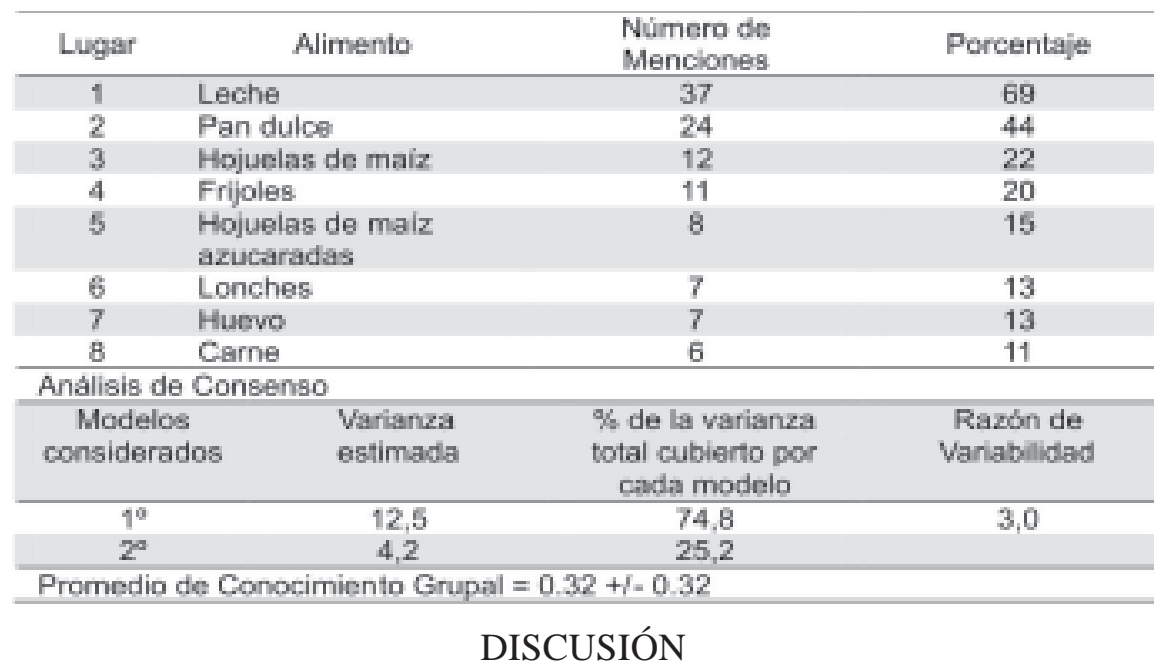

El análisis de consenso es una técnica para medir el conocimiento compartido de la competencia cultural sobre un dominio particular, al evidenciar el grado en que una serie de ítems señalados, forma la estructura modélica de un dominio culturalmente coherente para un grupo definido (16). Nuestro estudio acentúa el aspecto cultural del proceso nutricio, durante una etapa de la vida humana donde la vulnerabilidad del binomio madre-hijo, es importante. De acuerdo a nuestra perspectiva, la cognición nutricia (adquirida y limitada socialmente), esta representada como una estructura finita de ítems, que constituyen el acervo cultural de las personas, posibilita o no el desarrollo de aptitudes para lograr la salud física, mental y social de las adolescentes embarazadas.

Como se sabe cada método de encuesta dietética presenta ventajas y fallas que deben ser consideradas en función del objetivo a lograr, en nuestro estudio, el interés es cualitativo, es decir evidenciar la estructura del acuerdo grupal, lo cual representa una ventaja que se maximiza: el análisis de los tres tiempos de alimentación cotidiana a partir del consenso cultural de las protagonistas estudiadas, mediante la técnica de listas libres al captar la variabilidad de los ítems presentes en la cultura nutricia compartida por el grupo analizado, a diferencia de otros estudios donde se parte de recordatorios puntuales y de categorías estructuradas desde los investigadores y/o donde se hace tabula rasa de los alimentos consumidos en patrones generales (17), sin diferenciar los tiempos de alimentación y con el objetivo de cuantificar la ingestión dietética de individuos (18) sumados como grupo. Sin embargo, nuestro trabajo tiene también la 
limitante de no ser generalizable a toda la población de adolescentes embarazadas, por ser un estudio transversal y estar circunscrito a una población selecta de adolescentes embarazadas de bajo nivel económico que acude a un hospital público para población abierta.

De acuerdo con los resultados obtenidos en dos tiempos de la comida (desayuno y cena) se alcanza un alto nivel de acuerdo cultural, representados respectivamente por un solo modelo semántico de alimentos, en las adolescentes embarazadas que acuden a la consulta del HCG, lo cual interpretamos como la persistencia de la tradición de una alimentación mestiza, no sin cierta contaminación de alimentos industrializados de la dieta denominada "occidental" como son los cereales industrializados y el yogurt (19), fruto del proceso de globalización de bienes y servicios para la alimentación, que actualmente impacta a nuestra población de adolescentes en un poco más de $50 \%$ de las mismas y que en términos generales es también encontrado en otros países como Brasil, Corea y Grecia (17).

La comida es el tiempo de alimento más vulnerado, ya que no manifiesta acuerdo compartido entre las adolescentes, lo cual sugiere por un lado: el retroceso de la tradición alimentaria del modelo sopa-verdura-carne, frijoles y tortillas y por otra parte de la incorporación cognitiva de los productos chatarra, situación trascendente por sus efectos nocivos, como sería la dualidad: obesidad para la madre y la desnutrición para el niño, condiciones ambas que favorecen a la larga, la incidencia y la perpetuación de enfermedades tales como la diabetes mellitus tipo 2 y la hipertensión arterial (20).

Se considera que el consenso cultural, como proceso, puede encontrarse, desde un alto nivel (como sucede para el desayuno y la cena en las adolescentes), pasando por un nivel bajo (caracterizado por la incorporación de otras sub-culturas, dentro de una tradición), hasta llegar al disenso cultural (producto de las visiones de experiencias con diversos resultados que se traslapan con el nivel sub-cultural) y terminar en situaciones idiosincrásicas donde el acuerdo no existe y por lo tanto, no hay un dominio cultural, aunque cada persona puede tener su punto de vista muy particular.

Los resultados encontrados nos obligan a trabajar en las áreas de promoción y la educación de la salud en los niveles individual (clínico) y grupal (programas y políticas) para promover los elementos nutricios que constituyen una dieta cultural y económicamente accesible, suficiente y equilibrada sobre todo para el desayuno y la cena de las adolescentes embarazadas, que son los 
tiempos de alimentación donde existe un consenso, que puede ser aprovechado para diseñar intervenciones y guías con carácter sustentable, es decir más allá de la situación del embarazo de la adolescente, con el objeto de promover aptitudes y posturas favorables para mantener y mejorar la salud individual y prevenir patologías especificas como son el sobrepeso , obesidad, diabetes e hipertensión.

En el caso del tiempo de la comida, la situación a enfrentar es más difícil, por la avalancha de publicidad y problemas de tiempo, dinero y esfuerzo que influyen negativamente para que el grupo de adolescentes embarazadas establezca no tan solo un consenso cultural, sino también las conductas apropiadas sobre los elementos nutricios adecuados para la comida de mediodía, que representa todavía para muchos mexicanos el principal tiempo de comida.

Ejemplos de ellos son:

Los productos de consumo popular los cuales no difieren solo del embalaje, la publicidad y la distribución, si no también de la calidad que cambia con el precio; una misma corporación comercializa dos marcas con calidades y precios diferentes para cada segmento del mercado técnico, en ello se observa que hay una relación inversa entre contenido calórico y calidad, vg. las galletas, lácteos y pastas.

Y que en nuestra cultura el hombre hace sus tres comidas pero dispone de dinero para hacer colaciones fuera del hogar, la mujer no, además de que participan en una comida familiar, si acaso la cena (leche y pan) excluyéndose de los alimentos más nutritivos $(21,22)$.

Lo antes señalado deja claro que el acto de alimentarse para una adolescente embarazada, no es acción meramente mecánica si no bastante compleja, por lo que debe enfrentarse de manera integral y multidisciplinaria

\section{REFERENCIAS}

1. Trotter RT, Chensul JJ. Methods in applied Anthropology. In: Bernard HR. Handbook of methods in cultural anthropology. Walnut Creeck USA: Altamira Press; 1998. p. 708-12.

2. Bernard HR. Research methods in cultural anthropology. Newbury Park. USA: Sage publications; 1988. p. $110-25$.

3. Rommey KS, Weller SC, H Batchelder. Culture as consensus: a theory of culture and informant accuracy. American anthropologist; 1986; 81 (2): 313-38.

4. Bock PK. Rethinking psychological anthropology: continuity and change in the study of human 
action. 2d Ed. Illinois USA: Waveland Press. 1999; p 190-208.

5. D'Andrade R. The development of cognitive anthropology. Cambridge USA: Cambridge University Press; 1995; p. 182-217.

6. Ibarra-Colado J, Calderón MM. Rivas ME. Mortalidad prenatal, prematurez y peso bajo al nacimiento en el embarazo de la mujer adolescente en un hospital general. Bol Med Hosp. Infant Méx 2002; 59: 706-12.

7. Maddaleno M, Suárez OE. Situación social de los adolescentes y jóvenes en América Latina. En: Maddaleno M editores. La salud del adolescente y del joven. Washington, D.C. OPS; 1995. p. 70-84.

8. Casanueva E, Morales M. Nutrición del adolescente. En: Casanueva E, Kaufer-Horwitz M, Pérez-Lizaur AB, Arroyo P. Nutriología médica. $2^{\mathrm{a}}$ ed. México: Panamericana; 2001. p. 88-101.

9. Heald FP, Gong EJ. Diet, nutrition and adolescence. In: Shills ME, Olson JA, Shike M, Ross CA. editors. Modern nutrition in health and disease. $9^{\mathrm{a}}$ ed. Baltimore, Maryland: Williams and Wilkins; 1999. p. 857-67.

10. Sarría A, Moreno LA. Alimentación del adolescente. En: Bueno M, Sarría A, Pérez-González JM. Nutrición en pediatría. 2da ed. Majadahonda Madrid: Ergon; 2003. p. 207-16.

11. Casillas CL, Vargas L. Alimentación y nutrición en la adolescencia. Cuadernos de nutrición 1985; 8 (3): 17-24.

12. Eisenstein E. Nutrición y salud en la adolescencia. En: Maddaleno M. editores. La salud del adolescente y del joven. Washington, D.C: Organización Panamericana de la Salud; 1995. p. $144-57$.

13. Weller SC, Rommey AK. Systematic data collection qualitative research methods series 10. Newbury Park. USA: Sage publications; 1988.

14. Fleisher MS, Harrington JA. Freelisting. Management at a women's federal prision camp. In: De Munick VC, Sobo EJ, editors. Using methods in the field. USA: Altamira Press; 1998. p. 69-84.

15. Borgatti SP. Anthropac 4.0 Columbia Analityc Technologies; 1992.

16. Caulkins D. Consensus Analysis. Do Scottish business adviser agree on models of success? In: De Munick VC, Sobo EJ, editors. Using methods in the field. USA: Altamira Press; 1998. p. 179-96.

17. Lozada AL, Flores M, Rodríguez S, Barquera S. Patrones de dieta en adolescentes mexicanas. Salud Pública de México. 2007; 49 (4): 263-273.

18. Parra CS, Romieu I, Hernández AM, Madrigal FH. Usos y limitaciones de los métodos de encuesta dietética. En: Herlinda Madrigal Fritch y Homero Martínez Salgado. Manual de encuestas de dieta. Instituto de Salud Pública. 1996. p: 25-45.

19. Popkin BM. The nutrition transition: an overview of world patterns of change. Nut Rev 2004; (7Pt 2): S140-SN143.

20. Barker DJP, Osmond C, Law CM. The intrauterine and early postneonatal origins as a cardiovascular disease and chronic bronchitis. J. Epidemiology Community Health 1989; 43: $237-240$.

21. Aguirre P. Aspectos socio-antropológicos de la obesidad en la pobreza. En Manuel Peña y Jorge Bacallao. La obesidad en la pobreza: un nuevo reto para la salud pública. Organización Panamericana de la Salud. Publicación científica No 576. Washington. EUA; 2000. p. $13-25$.

22. Peña M, Bacallao J. La obesidad en la pobreza: un problema emergente en las Américas. En: Manuel Peña y Jorge Bacallao. La obesidad en la pobreza: un nuevo reto para la salud pública. Organización Panamericana de la Salud. Publicación científica No 576. Washington. EUA. 2000. p. 3-11. 